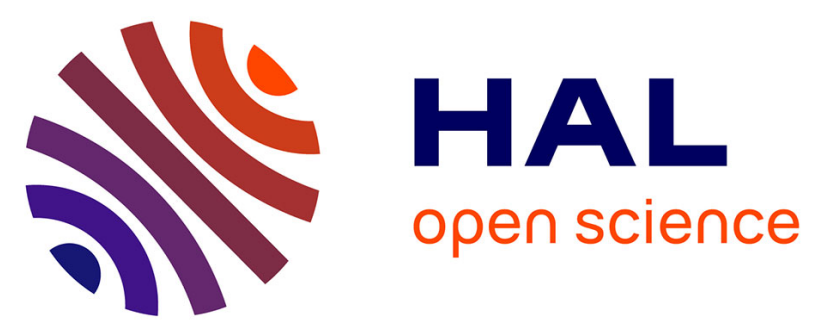

\title{
The Likelihood of the Consistency of Collective Rankings under Preferences Aggregation with Four Alternatives using Scoring Rules: A General Formula and the Optimal Decision Rule
}

Eric Kamwa, Vincent Merlin

\section{To cite this version:}

Eric Kamwa, Vincent Merlin. The Likelihood of the Consistency of Collective Rankings under Preferences Aggregation with Four Alternatives using Scoring Rules: A General Formula and the Optimal Decision Rule. Computational Economics, 2019, 53 (4), pp.1377-1395. 10.1007/s10614-018-9816-7 . hal-01757742

\author{
HAL Id: hal-01757742 \\ https://hal.univ-antilles.fr/hal-01757742
}

Submitted on 3 Apr 2018

HAL is a multi-disciplinary open access archive for the deposit and dissemination of scientific research documents, whether they are published or not. The documents may come from teaching and research institutions in France or abroad, or from public or private research centers.
L'archive ouverte pluridisciplinaire HAL, est destinée au dépôt et à la diffusion de documents scientifiques de niveau recherche, publiés ou non, émanant des établissements d'enseignement et de recherche français ou étrangers, des laboratoires publics ou privés. 
myjournal manuscript No.

(will be inserted by the editor)

\title{
The Likelihood of the Consistency of Collective Rankings under Preferences Aggregation with Four Alternatives using Scoring Rules: A General Formula and the Optimal Decision Rule
}

\author{
Eric Kamwa • Vincent Merlin
}

Received: date / Accepted: date

\begin{abstract}
In most of the social choice literature dealing with the computation of the exact probability of voting events under the impartial culture assumption, authors deal with no more than four constraints to describe voting events. With more than four constraints, most of the authors rely on Monte-Carlo simulations. It is usually more tricky to estimate the probability of events described by five constraints. Gehrlein and Fishburn (1980) have tried, but their conclusions are based on conjectures. In this paper, we circumvent this conjecture by having recourse to the technique suggested by Saari and Tataru (1999) in order to compute the limit probability of the consistency of collective rankings when there are four competing alternatives given that the decision rule is a scoring rule. We provide a general formula for the limit probability of the consistency and we determine the optimal decision rules among the scoring rules that provide the best guarantee of consistency. Given the collective ranking on a set $A$, we have consistency if the collective ranking on $B$ a proper subset of $A$ is not altered after some alternatives are removed from $A$.
\end{abstract}

Keywords Consistency $\cdot$ Scoring rules · Optimization · Impartial culture $\cdot$ Probability

Mathematics Subject Classification (2000) 91B14 $\cdot 91 \mathrm{~B} 12 \cdot 46 \mathrm{~N} 10$

E. Kamwa $(\bowtie)$

LC2S UMR CNRS 8053, Université des Antilles, Campus Schœlcher, Martinique

E-mail: eric.kamwa@univ-antilles.fr

V. Merlin

CNRS and CREM UMR CNRS 6211, Université de Caen Normandie

E-mail: vincent.merlin@unicaen.fr 


\section{Introduction}

One of the main objectives of social choice theory is the aggregation of individual preferences into a collective ranking that is the determination of a complete order over the set of the alternatives. Each agent (also called a "voter") is asked to provide a (complete) ranking of alternatives and a given voting or decision rule is used to determine the collective ranking (social outcome). The aggregation of preferences has wide applications in group decision making. In the framework of statistics, it is called the "consensus ranking problem" (see the seminal papers of Kendall (1938), Mallows (1957)). Scoring rules are among the most widely used decision rules; with a scoring rule, voters give points to candidates according to their rankings and the total number of points received by a candidate defines his scores for this rule. So, the alternative with the greatest (resp. with the lowest) score will appear at the top (resp. at the bottom) of the collective ranking and so on.

Sometimes, the aggregation of individual preferences can lead to counterintuitive outcomes called "voting paradoxes". For a non-exhaustive review of voting paradoxes, the reader can refer to Felsenthal (2012), Gehrlein (2006), Gehrlein and Lepelley (2010, 2017), Nurmi (1999). Researchers in the field of social choice theory not only describe or analyze voting paradoxes but also focus on their likelihood. They sometimes use probabilities as a criterion for comparing the decision rules; given a paradox, one voting rule is a better than another one if the likelihood of this paradox is less than that with the other rule. The Impartial Culture (IC) assumption is one amongst others, of the well-known hypotheses under which the computations are carried out. ${ }^{1}$ First introduced in the social choice literature by Gehrlein and Fishburn (1976), it is assumed that each agent (voter) chooses his preference (ranking) according to a uniform probability distribution. With $m$ alternatives $(m \geq 3)$, the probability of each of the $m$ ! possible strict rankings to being chosen independently is equal to $\frac{1}{m !}$ and the likelihood of a given voting situation ${ }^{2} \tilde{n}=\left(n_{1}, n_{2}, \ldots, n_{t}, \ldots, n_{m !}\right)$ is

$$
\operatorname{Prob}\left(\tilde{n}=\left(n_{1}, n_{2}, \ldots, n_{t}, \ldots, n_{m !}\right)\right)=\frac{n !}{\prod_{i=1}^{m !} n_{i} !} \times(m !)^{-n}
$$

with $n$ the size of the electorate and $n_{t}$ the number of agents with ranking of type $t$ among the $m$ ! possible rankings. For more details about the IC assumption, see among others Berg and Lepelley (1994), Gehrlein and Fishburn (1976), Gehrlein and Lepelley (2010, 2017).

According to Gehrlein (1979), one can derive the likelihood of most voting events under the IC assumption by using existing results on the representations of quadrivariate normal rules as suggested by Plackett (1954). ${ }^{3}$ Gehrlein-Fishburn's technique usually needs a good knowledge of the existing formulas in statistics for the representation of quadrivariate positive orthants (Abrahamson, 1964, Gehrlein, 1979). One advantage with this method is that it leads to compact formulas that help to determine which are the optimal decision rules given a voting event. In most of the social choice literature, when computing the exact probability of voting events under IC, authors tend to deal with events described by no more than four constraints (see Gehrlein and Lepelley $(2010,2017))$. With more than four constraints, most of the authors rely on Monte-Carlo simulations.

It is usually more tricky to estimate the probability of events described by five constraints and to determine the optimal decision rules. Gehrlein and Fishburn (1980) have tried, but their conclusions are based on conjectures. To be more precise, Gehrlein and Fishburn (1980) have analyzed what we call the "the consistency of collective ranking": going from a set $A$ with four alternatives and $B$ a proper subset of $A$ with three alternatives, they tried to evaluate for all the scoring rules, the mean limiting probability ${ }^{4}$ that a collective ranking on $A$ will be consistent with the collective ranking on $B$ when one element is removed from $A$. Gehrlein and Fishburn

\footnotetext{
1 For more on the other assumptions, please refer to Berg and Lepelley (1994), Gehrlein and Lepelley (2010).

2 We give a formal definition of a voting situation in Section 2.

${ }^{3}$ Assume $\left(X_{1}, X_{2}, \cdots, X_{n}\right)$ a vector of $n$ random variables with a nonsingular multivariate normal distribution. Plackett (1954) evaluated the probability $P\left(X_{1}>x_{1}, X_{2}>x_{2}, \cdots, X_{n}>x_{n}\right)$; he ended with a reduction formula of this probability based on the numerical quadrature for $n=3,4$.

4 A limiting probability is computed when the size of the electorate tends to infinity.
} 
(1980) were not able to say which scoring rules maximize or minimize this limiting probability: they simply concluded with a conjecture. In this paper, we follow a technique suggested by Saari and Tataru (1999) and we show how computations with five constraints can be applied to the framework of Gehrlein and Fishburn (1980), and we use some algorithms to find out which scoring rules maximize/minimize the limiting probability of consistency.

Independently from the works of Gehrlein (1979), Gehrlein and Fishburn (1976, 1980, 1981), Saari and Tataru (1999) suggested a geometric technique to derive the likelihood (under IC) of voting events described by four constraints. The Saari-Tataru's technique can be used without having recourse to the Plackett formulas and just needs good software to implement the procedure. The drawback is that formulas are not compact in general. However, as the Saari and Tataru (1999)'s technique is based upon the integration of a differential volume, a wise choice of the integration parameter can sometimes lead to compact formulas (Merlin et al., 2002, 2000, Tataru and Merlin, 1997). Fortunately, though the Gehrlein-Fishburn's technique and the Saari-Tataru's technique may lead to different formulas, their derivation lead to the same figures. Using the SaariTataru's technique, we obtain a general formula for the limiting probability of the consistency for all the scoring rules. Our approach can also be used in order to extend Gehrlein and Fishburn (1980)'s results to the following problem: given a set $A$ with four alternatives and $B$ a proper subset of $A$ with three alternatives, what is the probability to getting any of the rankings on the three-alternative subset? Given the general formula we obtain, we suggest a simple algorithm to determine the scoring rules that maximize/minimize the corresponding probability. So, using our algorithm based on optimization tools, one can then remove the Gehrlein-Fishburn's conjectures. Due to the fact that Saari-Tataru's technique leads to formulas that are not compact, we are not going to report all the general formulas here ${ }^{5}$. In four-alternative situations, we also highlight some important relations that one can use in order to derive the likelihood of other voting events ranging from that of a particular event. We also provide a generic MAPLE-sheet for our approach which can be used for computations of the likelihood of any four-alternative voting event described by five constraints when scoring rules are used ${ }^{6}$. First of all, let us present the basic definitions of the social choice literature.

\section{Basic framework of social choice theory}

\subsection{Preferences}

Let $N$ be the set of $n$ voters $(n \geq 2)$ and $A$ the set of $m$ alternatives, $m \geq 3$. The binary relation $R$ over $A$ is a subset of the Cartesian product $A \times A$. For $a, b \in A$, if $(a, b) \in R$, we write $a R b$ to say that " $a$ is at least good as $b$ ". $\neg a R b$ is the negation of $a R b$. If we have $a R b$ and $\neg b R a$, we will say that " $a$ is better or strictly preferred to $b$ ". In this case, we write $a P b$ with $P$ the asymmetric component of $R$. The preference profile $\pi=\left(P_{1}, P_{2}, \ldots, P_{i}, \ldots, P_{n}\right)$ gives all the linear orders ${ }^{7}$ of all the $n$ voters on $A$ where $P_{i}$ is the strict ranking of a given voter $i$ over $A$. When we consider the preference of voter $i$ on $B \subset A$, we will simply use the restriction of $P_{i}$ to $B$. The set of all the preference profiles of size $n$ on $A$ is denoted by $\mathcal{P}(A)^{n}$. A voting situation $\tilde{n}=\left(n_{1}, n_{2}, \ldots, n_{t}, \ldots, n_{m !}\right)$ indicates the number of voters for each linear order such that $\sum_{t=1}^{m !} n_{t}=n$. In the sequel, we simply write $\left(\begin{array}{l}a \\ b \\ c \\ d\end{array}\right)$ or $a b c d$ to say that $a$ is strictly preferred to $b, b$ strictly preferred to $c$ and $c$ strictly preferred to $d$. Table 1 gives the labels of all the 24 types of linear orders with four alternatives.

\footnotetext{
5 We provide a supplement document in which the reader can see how our formulas look .

6 The methodology developed in this paper was recently used and applied by Kamwa and Merlin (2015) to perform probability computations for some voting events under some popular voting rules.

7 A linear order is a binary relation that is transitive, complete and antisymmetric. The binary relation $R$ on $A$ is transitive if for $a, b, c \in A$, if $a R b$ and $b R c$ then $a R c$. $R$ is antisymmetric if for all for $a \neq b, a R b \Rightarrow \neg b R a$; if we have $a R b$ and $b R a$, then $a=b . R$ is complete if and only if for all $a, b \in A$, we have $a R b$ or $b R a$.
} 
Table 1 Labels of preferences on $A=\{a, b, c, d\}$

\begin{tabular}{|cccccccccccc|}
\hline$n_{1}$ & $n_{2}$ & $n_{3}$ & $n_{4}$ & $n_{5}$ & $n_{6}$ & $n_{7}$ & $n_{8}$ & $n_{9}$ & $n_{10}$ & $n_{11}$ & $n_{12}$ \\
\hline$a$ & $a$ & $a$ & $a$ & $a$ & $a$ & $b$ & $b$ & $b$ & $b$ & $b$ & $b$ \\
$b$ & $b$ & $c$ & $c$ & $d$ & $d$ & $a$ & $a$ & $c$ & $c$ & $d$ & $d$ \\
$c$ & $d$ & $b$ & $d$ & $b$ & $c$ & $c$ & $d$ & $a$ & $d$ & $a$ & $c$ \\
$d$ & $c$ & $d$ & $b$ & $c$ & $b$ & $d$ & $c$ & $d$ & $a$ & $c$ & $a$ \\
\hline \hline$n_{13}$ & $n_{14}$ & $n_{15}$ & $n_{16}$ & $n_{17}$ & $n_{18}$ & $n_{19}$ & $n_{20}$ & $n_{21}$ & $n_{22}$ & $n_{23}$ & $n_{24}$ \\
\hline$c$ & $c$ & $c$ & $c$ & $c$ & $c$ & $d$ & $d$ & $d$ & $d$ & $d$ & $d$ \\
$a$ & $a$ & $b$ & $b$ & $d$ & $d$ & $a$ & $a$ & $b$ & $b$ & $c$ & $c$ \\
$b$ & $d$ & $a$ & $d$ & $a$ & $b$ & $b$ & $c$ & $a$ & $c$ & $a$ & $b$ \\
$d$ & $b$ & $d$ & $a$ & $b$ & $a$ & $c$ & $b$ & $c$ & $a$ & $b$ & $a$ \\
\hline
\end{tabular}

\subsection{Scoring rules and consistency of collective rankings}

A scoring rule is a decision rule that gives points to alternatives according to the position they have in voters' rankings. We denote by $w=\left(w_{1}, w_{2}, w_{3}, \ldots, w_{j}, \ldots, w_{m}\right)$ the normalized scoring vector associated to the voting system such that $w_{1}=1 \geq w_{2} \geq \ldots \geq w_{j} \geq \ldots \geq w_{m}=0$. The most famous scoring rules are: the simple Plurality rule $\left(w_{2}=\ldots=w_{m}=0\right)$, the Antiplurality rule $\left(w_{2}=\ldots=w_{m-1}=1\right)$ and the Borda rule $\left(w_{j}=\frac{m-j}{m-1}\right)$.

The total number of points received by an alternative defines its score for the considered rule. Let $r(i, a, A)$ be the rank of alternative $a \in A$ in voter $i$ 's ranking, $r(i, a, A)=\sharp\left\{x \in A: x P_{i} a\right\}+1$. Given the voting system $w$, the total score of alternative $a \in A$ is given by $S(A, w, \pi, a)=$ $\sum_{i=1}^{n} w_{r(i, a, A)}$.

We denote by $R(A, w, \pi)$ (or simply $R(A, \pi)$ ) the collective ranking on $A$ given the profile $\pi$ for the scoring vector $w$. In $R(A, \pi)$, the alternative with the greatest total score appears at the top and the one with lowest total score is ranked last. For all $B \subseteq A$ with $|B| \geq 2$, we define $w^{B}$ the scoring vector of dimension $|B|$ used to rank alternatives in the subset $B$. As with $w$, we have $w^{B}=\left(w_{1}^{B}, w_{2}^{B}, \ldots, w_{\sharp B}^{B}\right)$ such that $w_{1}^{B}=1 \geq w_{2}^{B} \geq \ldots \geq w_{\sharp B}^{B}=0$. So, for a given subset $B$, the preference $P_{i}$ of voter $i$ is now defined by its restriction to $B$. Hence, given $w^{B}$, for an alternative $a \in B$, his score is given by $S(B, w, \pi, a)=\sum_{i=1}^{n} w_{r(i, a, B)}^{B}$. We denote by $R(\pi, B)$ the collective ranking on $B$ when the profile $\pi$ is restricted to $B$.

We denote by $R(\pi, A / B)$ the restriction of the collective ranking over $A$ on alternatives in $B$. When restricting the profile $\pi$ from $A$ to $B$, if $R(\pi, B)=R(\pi, A / B)$, the alternatives in the collective ranking on $B$ appear in the same order as in the collective ranking on $A$. In such a case, we will say that the scoring rule $w^{B}$ agrees or is consistent with the scoring rule $w$. If $R(\pi, B) \neq R(\pi, A / B)$, the alternatives in the collective ranking on $B$ do not appear in the same order as in the collective ranking on $A$. In a such case, we will say that the scoring rule $w^{B}$ is not consistent with the scoring rule $w$. Even worse, the alternatives in the collective ranking on $B$ may appear in the reverse order of that of the collective ranking on $A$.

In this paper we will focus on $|A|=4$ and $|B|=3$. The case $|A|=4$ and $|B|=2$ has been deeply analyzed by Gehrlein and Fishburn $(1980,1981)$. So, if $A=\{a, b, c, d\}$ then $B \in$ $\{\{a, b, c\},\{a, b, d\},\{a, c, d\},\{b, c, d\}\}$. Assuming that the same scoring rule is used on both the whole set and on the three alternative subsets, our analysis will focus on the whole family of scoring rules and we will use $w=(1, w, s, 0)$ on $A$ and $w^{B}=(1, z, 0)$ on $B$ with $0 \leq w \leq 1$, $0 \leq s \leq 1$ and $0 \leq z \leq 1$

\section{Generalization of probability computations of consistency}

\subsection{Methodology}

Our objective here is to generalize the evaluation of consistency probabilities under the IC assumption for $n$ large and for all the scoring vectors $w=(1, w, s, 0)$ on $A=\{a, b, c, d\}$ and $w^{B}=(1, z, 0)$ on $B=\{a, b, c\}$. 
With $A=\{a, b, c, d\}$, we denote by $P_{\infty}^{I C}\left(\left(w, w^{B}\right), a b c / a b c d\right)$ the limiting probability under IC such that the ranking $a b c$ occurs when $d$ the last ranked alternative of the collective ranking on $A$ is removed given the couple of tallies $\left(w, w^{B}\right)$. In the same way we define $P_{\infty}^{I C}\left(\left(w, w^{B}\right), a b c / a b d c\right)$, $P_{\infty}^{I C}\left(\left(w, w^{B}\right), a b c / a d b c\right), P_{\infty}^{I C}\left(\left(w, w^{B}\right), a b c / d a b c\right)$, and so on. One can notice that $P_{\infty}^{I C}\left(\left(w, w^{B}\right), a b c / a b c d\right)$ is the probability that the three-alternative collective ranking is consistent with the four-alternative collective ranking when $d$ is removed; while $P_{\infty}^{I C}\left(\left(w, w^{B}\right), c b a / a b c d\right)$ is the probability that the three-alternative collective ranking is totally the reverse of that with four alternatives after $d$ is removed.

In this section we will only focus on the probability computation of the event "abcd is the collective ranking with the scoring vector $(1, w, s, 0)$ and $a b c$ is the collective ranking with the scoring vector $(1, z, 0)$ when $d$ is removed". The calculations for all the other events and for all the other scoring rules follow the same scheme. So, with $A=\{a, b, c, d\}$ and $B=\{a, b, c\}$, our objective is to evaluate the probability of the event described by Equation (2) under the IC assumption, for $n$ large.

$$
\left\{\begin{array}{l}
S\left(A, w_{P}, \pi, a\right)>S\left(A, w_{P}, \pi, b\right) \\
S\left(A, w_{P}, \pi, b\right)>S\left(A, w_{P}, \pi, c\right) \\
S\left(A, w_{P}, \pi, c\right)>S\left(A, w_{P}, \pi, d\right) \\
S\left(B, w_{P}, \pi, a\right)>S\left(B, w_{P}, \pi, b\right) \\
S\left(B, w_{P}, \pi, b\right)>S\left(B, w_{P}, \pi, c\right)
\end{array}\right.
$$

We denote by $\vec{\Omega}$ a $24 \times 1$ matrix, $\vec{\Omega}=\left(\begin{array}{c}n_{1} \\ n_{2} \\ \vdots \\ n_{24}\end{array}\right)$. Given the labels of the 24 preference types for $m=4$ presented in Table 1 , we rewrite each of the equations as a product $\vec{v} \times \vec{\Omega}$ with $\vec{v}$ a $1 \times 24$ matrix.

$S\left(A, w_{P}, \pi, a\right)>S\left(A, w_{P}, \pi, b\right)$ is described by Equation 3 :

$(-w+1,-w+1,-s+1,1,-s+1,1,-1+w,-1+w,-1+s,-1,-1+s,-1,-s+w, w,-w+s,-w, s,-s,-s+w, w,-w+s,-w, s,-s) \vec{\Omega}>0$ $S\left(A, w_{P}, \pi, b\right)>S\left(A, w_{P}, \pi, c\right)$ is described by Equation 4 :

$(-s+w, w,-w+s,-w, s,-s,-s+1,1,-w+1,-w+1,1,-s+1,-1+s,-1,-1+w,-1+w,-1,-1+s, s,-s, w,-s+w,-w,-w+s) \vec{\Omega}>0$

$S\left(A, w_{P}, \pi, c\right)>S\left(A, w_{P}, \pi, d\right)$ is described by Equation 5 :

$(s,-s, w,-s+w,-w,-w+s, s,-s, w,-s+w,-w,-w+s, 1,-s+1,1,-s+1,-w+1,-w+1,-1,-1+s,-1,-1+s,-1+w,-1+w) \vec{\Omega}>0$

$S\left(B, w_{P}, \pi, a\right)>S\left(B, w_{P}, \pi, b\right)$ is described by Equation 6 :

$(-z+1,-z+1,1,1,-z+1,1,-1+z,-1+z,-1,-1,-1+z,-1, z, z,-z,-z, z,-z,-z+1,1,-1+z,-1, z,-z) \vec{\Omega}>0(6)$

$S\left(B, w_{P}, \pi, b\right)>S\left(B, w_{P}, \pi, c\right)$ is described by Equation 7 :

$(z, z,-z,-z, z,-z, 1,1,-z+1,-z+1,1,-z+1,-1,-1,-1+z,-1+z,-1,-1+z, z,-z, 1,-z+1,-1,-1+z) \vec{\Omega}>0(7)$

To make our calculations, we proceed in two steps. 
3.1.1 First step: Computation of the limit conditional probability of the event "abcd is the collective ranking and $S\left(B, w_{P}, \pi, a\right)>S\left(B, w_{P}, \pi, b\right)$ when $d$ is removed"

This event is described by Equations 3 to 6 . We rewrite Equation 6 by using a parameter $t$.

$$
\begin{array}{r}
(s+t(-z+1-s),-s+t(-z+1+s), w+t(1-w),-s+w+t(1+s-w),-w+t(-z+1+w), \\
-w+s+t(1+w-s), s+t(-1+z-s),-s+t(-1+z+s), w+t(-1-w),-s+w+t(-1-w+s), \\
-w+t(-1+z+w),-w+s+t(-1-s+w), 1+t(z-1),-s+1+t(z-1+s), 1+t(-z-1), \\
-s+1+t(-z-1+s),-w+1+t(z-1+w),-w+1+t(-z-1+w),-1+t(-z+2),-1+s+t(2-s), \\
-1+t(z),-1+s+t(-s),-1+w+t(z+1-w),-1+w+t(-z+1-w)) \vec{\Omega}>0
\end{array}
$$

When $t=0$, Equation 8 is equivalent to Equation 3. In $t=1$, it is fully equivalent to Equation 6. Our proof technique will in fact evaluate the probability that Equations (3),(4),(5) and (8) are satisfied under IC for $n$ large. In $t=0$, we recover the value $\frac{1}{24}$ which is the probability of $a b c d$ to being the collective ranking while in $t=1$ we will derive the limiting probability of the event described by Equations (3),(4),(5) and (8).

Given four alternatives, it is assumed under the Impartial Culture assumption that each voter is equally likely to have one of the 24 preference types. Let $x_{i}$ be the random variable that associates to each voter $i$ a 24-component vector with probability $\frac{1}{24}$ of having 1 in each position. The expectation of $x_{i}$ is

$$
E\left(x_{i}\right)=\left(\frac{1}{24}, \frac{1}{24}, \ldots, \frac{1}{24}\right)
$$

and the covariance matrix is a diagonal $24 \times 24$ matrix with the common entry $\sigma$ given by

$$
\sigma^{2}=E\left(x_{i}^{2}\right)-E\left(x_{i}\right)^{2}
$$

Let

$$
m^{T}=\left(m_{1}, m_{2}, \ldots m_{24}\right)^{T}=\frac{1}{\sigma \sqrt{n}}\left[\left(\begin{array}{c}
n_{1} \\
\vdots \\
n_{24}
\end{array}\right)-\left(\begin{array}{c}
\frac{n}{24} \\
\vdots \\
\frac{n}{24}
\end{array}\right)\right]
$$

The Central Limit Theorem in $\mathbb{R}^{23}$ implies

$$
\mu\left[m^{T}\right] \mapsto \frac{1}{(\sqrt{2} \pi)^{23}} e^{\frac{-|t|^{2}}{2}} \lambda
$$

as $n \rightarrow \infty$ where $t=\left(t_{1}, t_{2}, \ldots, t_{24}\right) \in \mathbb{R}^{24},|t|^{2}=t_{1}^{2}+\cdots+t_{24}^{2}$ and $\lambda$ is the Lebesgue measure on the 23-dimensional hyperplane $t_{1}+\cdots+t_{24}=0$. Note that since $m^{T}$ has the measure supported on the hyperplane $m_{1}+\cdots+m_{24}=0$, the limit of $m^{T}$ as $n \rightarrow \infty$ is also a measure supported on $t_{1}+\cdots+t_{24}=0$. To compute the probability that $a b c d$ is the collective ranking and $S\left(B, w_{P}, \pi, a\right)>$ $S\left(B, w_{P}, \pi, b\right)$ when $d$ is removed, we need to evaluate the probability that a voting situation is characterized by the inequalities $(3),(4),(5)$ and $(8) ; m$ satisfies inequalities $(3),(4),(5)$ and (8) if and only if $\tilde{n}=\left(n_{1}, n_{2}, \ldots, n_{24}\right)$ also satisfies them. Then, by the Central Limit Theorem, we write

$$
\operatorname{Pr}\left(m^{T} \text { satisfies }(3),(4),(5) \text { and }(8)\right) \mapsto \frac{1}{(\sqrt{2} \pi)^{23}} \int_{C_{1}} e^{\frac{-|t|^{2}}{2}} d \lambda
$$

where $C_{1}=\left\{t \in \mathbb{R}^{24}, t\right.$ satisfies $\left((3),(4),(5)\right.$ and (8)and $\left.\sum_{i=1}^{24}\left(t_{i}\right)=0\right\}$.

As the measure $\bar{\mu} \equiv \frac{1}{(\sqrt{2} \pi)^{23}} e^{\frac{-|t|^{2}}{2}} \lambda$ is absolutely continuous and radially symmetric, computing $\frac{1}{(\sqrt{2} \pi)^{23}} \int_{C_{1}} e^{\frac{-|t|^{2}}{2}} d \lambda$ reduces to finding the measure $\bar{\mu}$ of the cone $C_{1}$, when the measure is invariant 
to rotations. The measure $\bar{\mu}$ of such a cone is proportional to the Euclidean measure of the cone, that is, the measure on the sphere.

Saari and Tataru (1999) have developed a method of computing the probabilities of voting events under the Impartial culture. Some refinements of this method are done in Merlin et al. (2002, 2000), Merlin and Valognes (2004). This method is mainly based on linear algebra and the calculation of a differential volume in a spherical simplex of dimension $\nu$ using the Schläfli formula (see Coxeter (1935), Kellerhals (1989), Milnor (1982), Schläfli (1950)). This formula is given by:

$$
\operatorname{dvol}_{\nu}\left(C_{1}\right)=\frac{1}{(\nu-1)} \sum_{0 \leq j<k \leq \nu} \operatorname{vol}_{\nu-2}\left(S_{j} \cap S_{k}\right) d \alpha_{j k} ; \quad \operatorname{vol}_{0}=1
$$

with $\alpha_{j k}$ the dihedral angle formed by the facets $S_{j}$ and $S_{k}$ of the cone $C_{1}$. Following the arguments given by Saari and Tataru (1999), the probability that the inequalities are met simultaneously for a voting situation when $p_{i}=\frac{1}{24}, i=1, \ldots, 24$ for $n$ large is equal to the surface of the spherical simplex $T$ described by Equations $(3),(4),(5),(8)$ on the surface of the unit sphere in $\mathbb{R}^{4}$, divided by the surface of this sphere.

More precisely, if we denote by $P_{\infty}^{I C}\left(\left(w, w^{B}\right), a b c d-a b\right)$ the limit probability that abcd is the collective ranking and $S\left(B, w_{P}, \pi, a\right)>S\left(B, w_{P}, \pi, b\right)$ when $d$ is removed, we will derive

$$
P_{\infty}^{I C}\left(\left(w, w^{B}\right), a b c d-a b\right)=1+\frac{24}{\omega^{4}} \int_{0}^{t} d v_{\nu}\left(C_{1}\right) d t
$$

where $\omega^{4}=2 \pi^{2}$ is the volume of the unit sphere in $\mathbb{R}^{4}$.

Given the cone $C_{1}$, let $S_{1}$ be the facet defined by the Equation (3), $S_{2}$ the facet defined by the Equation (4), $S_{3}$ the facet defined by the Equation (5) and $S_{4}$ the facet defined by the Equation (8). Let $\vec{v}_{1}, \vec{v}_{2}, \vec{v}_{3}$ and $\vec{v}_{4}$ be the normal vectors to the hyperplanes $S_{1}, S_{2}, S_{3}$ and $S_{4}$ respectively.

$$
\begin{aligned}
& \overrightarrow{v_{1}}=(-w+1,-w+1,-s+1,1,-s+1,1,-1+w,-1+w,-1+s,-1,-1+s,-1,-s,-s+w, w,-w+s,-w, s,-s) \\
& \overrightarrow{v_{2}}=(-s+w, w,-w+s,-w, s,-s,-s+1,1,-w+1,-w+1,1,-s+1,-1+s,-1,-1+w,-1+w,-1,-1+s, s, \\
& -s, w,-s+w,-w,-w+s) \\
& \overrightarrow{v_{3}}=\quad(s,-s, w,-s+w,-w,-w+s, s,-s, w,-s+w,-w,-w+s, 1,-s+1,1,-s+1,-w+1,-w+1,-1,-1+s,-1, \\
& -1+s,-1+w,-1+w) \\
& \begin{aligned}
\overrightarrow{v_{4}}= & (s+t(-z+1-s),-s+t(-z+1+s), w+t(1-w),-s+w+t(1+s-w),-w+t(-z+1+w),-w+s+t(1+w-s), \\
& s+t(-1+z-s),-s+t(-1+z+s), w+t(-1-w),-s+w+t(-1-w+s),-w+t(-1+z+w),-w+s+t(-1-s+w),
\end{aligned} \\
& \begin{array}{c}
s+t(z-1),-s+1+t(z-1+s), 1+t(-z-1),-s+1+t(-z-1+s),-w+1+t(z-1+w),-w+1+t(-z-1+w),
\end{array} \\
& -1+t(-z+2),-1+s+t(2-s),-1+t(z),-1+s+t(-s),-1+w+t(z+1-w),-1+w+t(-z+1-w))
\end{aligned}
$$

Since $\overrightarrow{v_{j}}$ and $\overrightarrow{v_{k}}$ are respectively normal to $S_{j}$ and $S_{k}$, we can use the relationship

$$
\cos \left(\alpha_{j k}\right)=\frac{-\overrightarrow{v_{j}} \cdot \overrightarrow{v_{k}}}{\left\|\overrightarrow{v_{j}}\right\| \cdot\left\|\overrightarrow{v_{k}}\right\|}
$$

to derive the value of the dihedral angle $\alpha_{j k}$ between vectors $\overrightarrow{v_{j}}$ and $\overrightarrow{v_{k}}$. So, we compute $\alpha_{12}$, $\alpha_{13}, \alpha_{14}, \alpha_{23}, \alpha_{24}$ and $\alpha_{34}$. Therefore, we compute the differential angles $d \alpha_{j k}$. The vectors $\overrightarrow{v_{1}}$, $\overrightarrow{v_{2}}, \overrightarrow{v_{3}}$ and $\overrightarrow{v_{4}}$ lie in a 4-dimensional space. We find 19 other vectors $\left(\overrightarrow{v_{5}}\right.$ to $\left.\overrightarrow{v_{24}}\right)$ such that we get a basis for the orthogonal subspace. Then, we can calculate the vertexes $P_{123}=S_{1} \cap S_{2} \cap S_{3}$, $P_{124}=S_{1} \cap S_{2} \cap S_{4}, P_{134}=S_{1} \cap S_{3} \cap S_{4}$ and $P_{2345}=S_{2} \cap S_{3} \cap S_{4}$ by solving each of the systems described in Equation 9. 


$$
P_{123}:\left\{\begin{array}{l}
S_{1}=0 \\
S_{2}=0 \\
S_{3}=0 \\
S_{4}>0 \\
S_{5}=0 \\
S_{6}=0 \\
S_{7}=0 \\
\vdots \\
\vdots \\
S_{23}=0 \\
S_{24}=0
\end{array} \quad P_{124}:\left\{\begin{array}{l}
S_{1}=0 \\
S_{2}=0 \\
S_{3}>0 \\
S_{4}=0 \\
S_{5}=0 \\
S_{6}=0 \\
S_{7}=0 \\
\vdots \\
\vdots \\
S_{23}=0 \\
S_{24}=0
\end{array} \quad P_{134}:\left\{\begin{array}{l}
S_{1}=0 \\
S_{2}>0 \\
S_{3}=0 \\
S_{4}=0 \\
S_{5}=0 \\
S_{6}=0 \\
S_{7}=0 \\
\vdots \\
\vdots \\
S_{23}=0 \\
S_{24}=0
\end{array} \quad P_{234}:\left\{\begin{array}{l}
S_{1}>0 \\
S_{2}>0 \\
S_{3}=0 \\
S_{4}=0 \\
S_{5}=0 \\
S_{6}=0 \\
S_{7}=0 \\
\vdots \\
\vdots \\
\\
S_{23}=0 \\
S_{24}=0
\end{array}\right.\right.\right.\right.
$$

The solutions of these systems are four vertices: $P_{123}, P_{124}, P_{134}$ and $P_{234}$. Knowing these vertices, we are able to compute the following volumes

$$
\begin{aligned}
& \operatorname{vol}\left(S_{1} \cap S_{2}\right)=\measuredangle\left(P_{123}, P_{124}\right) \\
& \operatorname{vol}\left(S_{1} \cap S_{3}\right)=\measuredangle\left(P_{123}, P_{134}\right) \\
& \operatorname{vol}\left(S_{1} \cap S_{4}\right)=\measuredangle\left(P_{124}, P_{134}\right) \\
& \operatorname{vol}\left(S_{2} \cap S_{3}\right)=\measuredangle\left(P_{123}, P_{234}\right) \\
& \operatorname{vol}\left(S_{2} \cap S_{4}\right)=\measuredangle\left(P_{124}, P_{234}\right) \\
& \operatorname{vol}\left(S_{3} \cap S_{4}\right)=\measuredangle\left(P_{134}, P_{234}\right)
\end{aligned}
$$

It follows from the Schläfli's formula that,

$$
\begin{aligned}
& \operatorname{dvol}\left(C_{1}\right)=\operatorname{vol}\left(S_{1} \cap S_{2}\right) d \alpha_{12}+\operatorname{vol}\left(S_{1} \cap S_{3}\right) d \alpha_{13}+\operatorname{vol}\left(S_{1} \cap S_{4}\right) d \alpha_{14} \\
& \quad+\operatorname{vol}\left(S_{2} \cap S_{3}\right) d \alpha_{23}+\operatorname{vol}\left(S_{2} \cap S_{4}\right) d \alpha_{24}+\operatorname{vol}\left(S_{3} \cap S_{4}\right) d \alpha_{34}
\end{aligned}
$$

We have to multiply $\operatorname{dvol}\left(C_{1}\right)$ by 24 then divide it by 2 (since $\nu=3$ ) and then by $2 \pi^{2}$ (the volume of the unit sphere in $\left.\mathbb{R}^{4}\right)$ in order to obtain the final differential volume $\frac{24}{4 \pi^{2}} \int_{0}^{t} d v o l\left(C_{1}\right) d t$. We then derive at $t=1$ the value of the probability that abcd is the collective ranking and $S\left(B, w_{P}, \pi, a\right)>S\left(B, w_{P}, \pi, b\right)$ when $d$ is removed

$$
P_{\infty}^{I C}\left(\left(w, w^{B}\right), a b c d-a b\right)=1+\frac{6}{\pi^{2}} \int_{0}^{1} d v o l\left(C_{1}\right) d t
$$

We can now move to the second step where we will derive our consistency probability between $a b c d$ and $a b c$ when $d$ is removed.

\subsubsection{Second step: the generalized consistency probability}

Using the Schläfli formula again, the consistency probability we are looking for will be obtained by adding to $P^{I C}\left(\left(w, w^{B}\right), a b c d-a b\right)$, the integral of the differential volume characterized by the inequalities $(3),(4),(5),(6)$ and $(7)$. To determine this differential volume, we need to rewrite inequality (7) using the parameter $t$.

$$
\begin{gathered}
(-z+1+t(z+z-1),-z+1+t(z+z-1), 1+t(-z-1), 1+t(-z-1),-z+1+t(z-1+z), 1+t(-z-1),(10) \\
-1+z+t(-z+2),-1+z+t(-z+2),-1+t(-z+2),-1+t(-z+2),-1+z+t(-z+2),-1+t(-z+2) \\
z+t(-z-1), z+t(-z-1),-z+t(2 z-1),-z+t(2 z-1), z+t(-z-1),-z+t(2 z-1) \\
-z+1+t(2 z-1), 1+t(-z-1),-1+z+t(-z+2),-1+t(-z+2), z+t(-z-1),-z+t(2 z-1)) \vec{\Omega}>0
\end{gathered}
$$

When $t=0$, Equation (10) is equivalent to Equation (4). In $t=1$, it fully describes the situation of Equation (2). As in the first step, our proof technique will be based on the same arguments and will evaluate the probability that Equations $(3),(4),(5),(6)$ and (10) are satisfied 
under IC for $n$ large. In $t=0$, we recover the value $P_{\infty}^{I C}\left(\left(w, w^{B}\right), a b c d-a b\right)$ while in $t=1$ we will derive the probability of the event described by Equations (3),(4),(5),(6) and (10).

By the Central Limit Theorem, we can write

$$
\operatorname{Pr}\left(m^{T} \text { satisfies }(3),(4),(5),(6) \text { and }(10)\right) \mapsto \frac{1}{(\sqrt{2} \pi)^{23}} \int_{C_{2}} e^{\frac{-|t|^{2}}{2}} d \lambda
$$

where $C_{2}=\left\{t \in \mathbb{R}^{24}, t\right.$ satisfies $\left((3),(4),(5),(6)\right.$ and $(10)$; and $\left.\sum_{i=1}^{24}\left(t_{i}\right)=0\right\}$. Using the Schläfli formula

$$
\operatorname{dvol}_{\rho}\left(C_{2}\right)=\frac{1}{(\rho-1)} \sum_{0 \leq j<k \leq \rho} \operatorname{vol}_{\rho-2}\left(T_{j} \cap T_{k}\right) d \theta_{j k} ; \quad \operatorname{vol}_{0}=1
$$

Following the arguments stated by Saari and Tataru (1999), the probability that inequalities $(3),(4),(5),(6)$ and $(10)$ are met simultaneously for a voting situation when $p_{i}=\frac{1}{24}, i=1, \ldots, 24$ for $n$ large is equal to the surface of the spherical simplex $T$ described by Equations $(3),(4),(5)$, (8) on the surface of the unit sphere in $\mathbb{R}^{5}$, divided by the surface of this sphere. More precisely, we will derive

$$
P_{\infty}^{I C}\left(\left(w_{P}, w_{P}^{B}\right), a b c / a b c d\right)=P_{\infty}^{I C}\left(\left(w, w^{B}\right), a b c d-a b\right)+\frac{24}{\omega^{5}} \int_{0}^{t} d v o l_{\rho}\left(C_{2}\right) d t
$$

where $\omega^{5}=\frac{8 \pi^{2}}{3}$ is the volume of the surface of the unit sphere in $\mathbb{R}^{5}$.

Given the cone $C_{2}$, let $T_{1}$ be the facet defined by the Equation (3), $T_{2}$ the facet defined by the Equation (4), $T_{3}$ the facet defined by the Equation (5), $T_{4}$ the facet defined by the Equation (6) and $T_{5}$ the facet defined by the Equation (10). Let $\vec{s}_{1}, \vec{s}_{2}, \vec{s}_{3}, \vec{s}_{4}, \vec{s}_{5}$ be the normal vectors to the hyperplanes $T_{1}, T_{2}, T_{3}, T_{4}, T_{5}$.

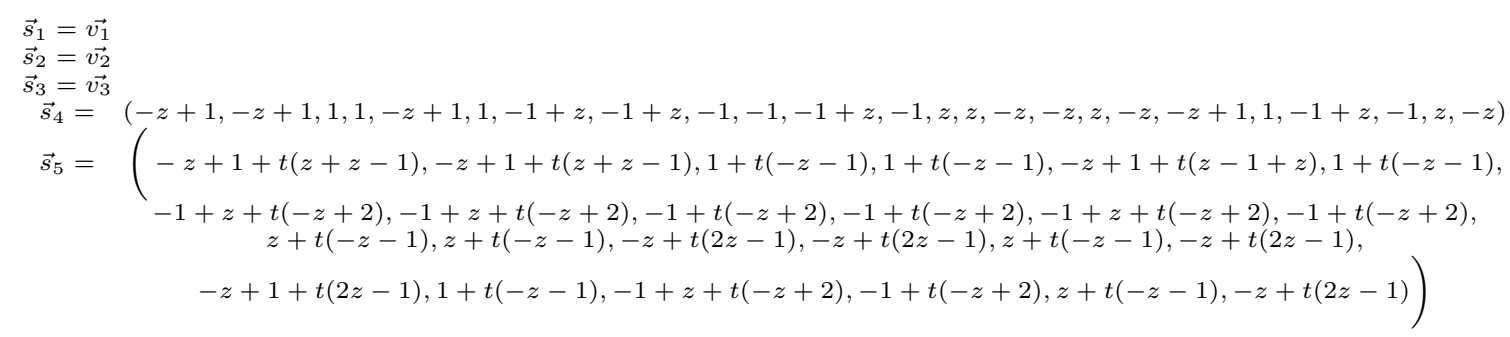

We then have the dihedral angles $\theta_{j k}$ between vectors $\overrightarrow{s_{j}}$ and $\overrightarrow{s_{k}}: \theta_{14}, \theta_{34}, \theta_{15}, \theta_{35}, \theta_{24}, \theta_{25}$ and $\theta_{45}$. Then, we compute the differential angles $d \theta_{14}, d \theta_{24}, d \theta_{34}, d \theta_{15}, d \theta_{35}, d \alpha_{24}, d \theta_{25}, d \alpha_{14}$ and $d \theta_{45}$. The vectors $\overrightarrow{s_{1}}, \overrightarrow{s_{2}}, \overrightarrow{s_{3}}, \overrightarrow{s_{4}}$ and $\overrightarrow{s_{5}}$ lie in a 4-dimensional space. We find 19 other vectors $\left(\overrightarrow{s_{6}}\right.$ to $\left.\overrightarrow{s_{24}}\right)$ to form a basis for the orthogonal subspace. Then, we calculate the vertexes $P_{1234}=T_{1} \cap T_{2} \cap T_{3} \cap T_{4}$, $P_{1235}=T_{1} \cap T_{2} \cap T_{3} \cap T_{5}, P_{1245}=T_{1} \cap T_{2} \cap T_{4} \cap T_{5}, P_{1345}=T_{1} \cap T_{3} \cap T_{4} \cap T_{5}$ and $P_{2345}=T_{2} \cap T_{3} \cap T_{4} \cap T_{5}$ by solving the following systems

$P_{1234}:\left\{\begin{array}{l}T_{1}=0 \\ T_{2}=0 \\ T_{3}=0 \\ T_{4}=0 \\ T_{5}>0 \\ T_{6}=0 \\ T_{7}=0 \\ \vdots \\ \vdots \\ T_{23}=0 \\ T_{24}=0\end{array} \quad P_{1235}:\left\{\begin{array}{l}T_{1}=0 \\ T_{2}=0 \\ T_{3}=0 \\ T_{4}>0 \\ T_{5}=0 \\ T_{6}=0 \\ T_{7}=0 \\ \vdots \\ \vdots \\ T_{23}=0 \\ T_{24}=0\end{array} \quad P_{1245}:\left\{\begin{array}{l}T_{1}=0 \\ T_{2}=0 \\ T_{3}>0 \\ T_{4}=0 \\ T_{5}=0 \\ T_{6}=0 \\ T_{7}=0 \\ \vdots \\ \vdots \\ T_{23}=0 \\ T_{24}=0\end{array} \quad P_{1345}:\left\{\begin{array}{l}T_{1}=0 \\ T_{2}>0 \\ T_{3}=0 \\ T_{4}=0 \\ T_{5}=0 \\ T_{6}=0 \\ T_{7}=0 \\ \vdots \\ \vdots \\ T_{23}=0 \\ T_{24}=0\end{array} \quad P_{2345}:\left\{\begin{array}{l}T_{1}>0 \\ T_{2}=0 \\ T_{3}=0 \\ T_{4}=0 \\ T_{5}=0 \\ T_{6}=0 \\ T_{7}=0 \\ \vdots \\ \vdots \\ T_{23}=0 \\ T_{24}=0\end{array}\right.\right.\right.\right.\right.$ 
Table 2 volumes and directions

\begin{tabular}{cc}
\hline volumes & Directions \\
\hline$\left(T_{1} \cap T_{2}\right)$ & $P_{1235}, P_{1235}, P_{1245}$ \\
$\left(T_{1} \cap T_{3}\right)$ & $P_{1234}, P_{1235}, P_{1345}$ \\
$\left(T_{1} \cap T_{4}\right)$ & $P_{1234}, P_{1245}, P_{1345}$ \\
$\left(T_{1} \cap T_{5}\right)$ & $P_{1235}, P_{1245}, P_{1345}$ \\
$\left(T_{2} \cap T_{3}\right)$ & $P_{1234}, P_{1235}, P_{2345}$ \\
$\left(T_{2} \cap T_{4}\right)$ & $P_{1234}, P_{1245}, P_{2345}$ \\
$\left(T_{2} \cap T_{5}\right)$ & $P_{1235}, P_{1245}, P_{2345}$ \\
$\left(T_{3} \cap T_{4}\right)$ & $P_{1234}, P_{1345}, P_{2345}$ \\
$\left(T_{3} \cap T_{5}\right)$ & $P_{1235}, P_{1345}, P_{2345}$ \\
$\left(T_{4} \cap T_{5}\right)$ & $P_{1245}, P_{1345}, P_{2345}$ \\
\hline \hline
\end{tabular}

The solutions of these systems are the five vertices: $P_{1234}, P_{1235}, P_{1245}, P_{1345}$ and $P_{2345}$. Knowing these vertices, we are able to compute the volumes $\left(T_{j} \cap T_{k}\right)$. Each of these volumes is the area of a triangle on the sphere in $\mathbb{R}^{3}$ defined by some directions. Table 2 gives the directions for each of these volumes.

Let us consider the volume $\left(T_{1} \cap T_{5}\right)$. By the Gauss-Bonnet theorem, the area of the triangle on the sphere in $R^{3}$ defined by directions $P_{1235}, P_{1245}$ and $P_{1345}$ is equal to the sum of the angles on the surface of the triangle minus $\pi$. We denote by $\gamma_{1235}, \gamma_{1245}$ and $\gamma_{1345}$ the angles on the surface of the triangle respectively defined at the vertexes $P_{1235}, P_{1245}$ and $P_{1345}$. Also, we define the angles $\delta_{1}=P_{1235, P_{1245}}, \delta_{2}=P_{1235, P_{1345}}$ and $\delta_{3}=P_{1245, P_{1345}}$. By applying the the Gauss-Bonnet formula, we have

$$
\begin{aligned}
\cos \left(\gamma_{1345}\right) & =\frac{\cos \left(\delta_{1}\right)-\cos \left(\delta_{2}\right) \cos \left(\delta_{3}\right)}{\sin \left(\delta_{2}\right) \sin \left(\delta_{3}\right)} \\
\cos \left(\gamma_{1245}\right) & =\frac{\cos \left(\delta_{2}\right)-\cos \left(\delta_{1}\right) \cos \left(\delta_{3}\right)}{\sin \left(\delta_{1}\right) \sin \left(\delta_{3}\right)} \\
\cos \left(\gamma_{1235}\right) & =\frac{\cos \left(\delta_{3}\right)-\cos \left(\delta_{1}\right) \cos \left(\delta_{2}\right)}{\sin \left(\delta_{1}\right) \sin \left(\delta_{2}\right)}
\end{aligned}
$$

and,

$$
\begin{aligned}
& \operatorname{vol}\left(T_{1} \cap T_{2}\right)=\gamma_{1234}+\gamma_{1235}+\gamma_{1245}-\pi \\
& \operatorname{vol}\left(T_{1} \cap T_{3}\right)=\gamma_{1234}+\gamma_{1235}+\gamma_{1345}-\pi \\
& \operatorname{vol}\left(T_{1} \cap T_{4}\right)=\gamma_{1234}+\gamma_{1245}+\gamma_{1345}-\pi \\
& \operatorname{vol}\left(T_{1} \cap T_{5}\right)=\gamma_{1235}+\gamma_{1245}+\gamma_{1345}-\pi \\
& \operatorname{vol}\left(T_{2} \cap T_{3}\right)=\gamma_{1234}+\gamma_{1235}+\gamma_{2345}-\pi \\
& \operatorname{vol}\left(T_{2} \cap T_{4}\right)=\gamma_{1234}+\gamma_{1245}+\gamma_{2345}-\pi \\
& \operatorname{vol}\left(T_{2} \cap T_{5}\right)=\gamma_{1235}+\gamma_{1245}+\gamma_{2345}-\pi \\
& \operatorname{vol}\left(T_{3} \cap T_{4}\right)=\gamma_{1234}+\gamma_{1345}+\gamma_{2345}-\pi \\
& \operatorname{vol}\left(T_{3} \cap T_{5}\right)=\gamma_{1235}+\gamma_{1345}+\gamma_{2345}-\pi \\
& \operatorname{vol}\left(T_{4} \cap T_{5}\right)=\gamma_{1245}+\gamma_{1345}+\gamma_{2345}-\pi
\end{aligned}
$$

Applying the Schläfli formula we get,

$$
\begin{aligned}
\operatorname{dvol}\left(C_{2}\right)=\operatorname{vol} & \left(T_{1} \cap T_{2}\right) d \theta_{12}+\operatorname{vol}\left(T_{1} \cap T_{3}\right) d \theta_{13}+\operatorname{vol}\left(T_{1} \cap T_{4}\right) d \theta_{14}+\operatorname{vol}\left(T_{1} \cap T_{5}\right) d \theta_{15} \\
& +\operatorname{vol}\left(T_{2} \cap T_{3}\right) d \theta_{23}+\operatorname{vol}\left(T_{2} \cap T_{4}\right) d \theta_{24}+\operatorname{vol}\left(T_{2} \cap T_{5}\right) d \theta_{25}+\operatorname{vol}\left(T_{3} \cap T_{4}\right) d \theta_{34} \\
& +\operatorname{vol}\left(T_{3} \cap T_{5}\right) d \theta_{35}+\operatorname{vol}\left(T_{4} \cap T_{5}\right) d \theta_{45}
\end{aligned}
$$


We then multiply $\operatorname{dvol}\left(C_{2}\right)$ by 24 and then divide it by $\frac{8 \pi^{2}}{3}$ (the volume of the hypersphere in $\mathbb{R}^{5}$ ) and then by 3 (since $\left.\rho=4\right)$ to obtain the final differential volume $\frac{3}{\pi^{2}} \int_{0}^{t} d v o l\left(C_{2}\right) d t$. At $t=1$ we then derive,

$$
\begin{aligned}
P_{\infty}^{I C}\left(\left(w, w^{B}\right), a b c / a b c d\right) & =P_{\infty}^{I C}\left(\left(w, w^{B}\right), a b c d-a b\right)+\frac{3}{\pi^{2}} \int_{0}^{1} d v o l\left(C_{2}\right) d t \\
& =1+\frac{6}{\pi^{2}} \int_{0}^{1} d \operatorname{vol}\left(C_{1}\right) d t+\frac{3}{\pi^{2}} \int_{0}^{1} d \operatorname{vol}\left(C_{2}\right) d t \\
& =1+\frac{3}{\pi^{2}} \int_{0}^{1}\left(2 d \operatorname{vol}\left(C_{1}\right)+\operatorname{dvol}\left(C_{2}\right)\right) d t
\end{aligned}
$$

Following the same scheme, we can obtain one general formula for each four-alternative event described by five constraints. The formulas we obtain are quite huge; as said before, this is due to the fact that the Saari-Tataru's technique does not always lead to compact formulas. ${ }^{8}$ So we are not going to report the formulas here; instead we provide a link (in the Appendix) where the reader can see how they look. Also in the Appendix, we provide a link to the generic MAPLE-sheet we used in order to perform our computations on consistency. Recall that this MAPLE-sheet can be used to compute the likelihood of any four-alternative voting events described by five constraints when scoring rules are used.

\subsection{Some numerical results}

We can now use our Maple-sheet in order to get some numerical results of consistency probabilities. In Table 3, we report the values of $P_{\infty}^{I C}\left(\left(w, w^{B}\right), a b c / \bullet\right)$, the limit probability that given the collective ranking on $A=\{a, b, c, d\}$ we will get the same collective ranking on $B=\{a, b, c\}$ when $d$ is removed. So, we consider the cases where $d$ is ranked first, second, third and last. We restrict our computations to some values of $w, s$ and $z$ in order to focus on the well-known scoring rules in social choice theory. The key considerations derive from Table 3 and this is also what we get

Table 3 Consistency probabilities $P_{\infty}^{I C}\left(\left(w, w^{B}\right), a b c / \bullet\right)$ for some values of $(w, s, z)$

\begin{tabular}{c||c||c||c||c||c||c||}
$(w, s, z)$ & $\left(\frac{2}{3}, \frac{1}{3}, \frac{1}{2}\right)$ & $(1,0,0)$ & $\left(1, \frac{1}{3}, 0\right)$ & $(1,1,1)$ & $\left(1, \frac{2}{3}, 1\right)$ & $(1,0,1)$ \\
\hline \hline$P_{\infty}^{I C}\left(\left(w, w^{B}\right), a b c / a b c d\right)$ & 0.719518 & 0.516969 & 0.594543 & 0.516969 & 0.594543 & 0.412089 \\
\hline$P_{\infty}^{I C}\left(\left(w, w^{B}\right), a b c / a b d c\right)$ & 0.840425 & 0.661087 & 0.737973 & 0.661087 & 0.737973 & 0.537710 \\
\hline$P_{\infty}^{I C}\left(\left(w, w^{B}\right), a b c / a d b c\right)$ & 0.840425 & 0.661087 & 0.737973 & 0.661087 & 0.737973 & 0.537710 \\
\hline$P_{\infty}^{I C}\left(\left(w, w^{B}\right), a b c / d a b c\right)$ & 0.719518 & 0.516969 & 0.594543 & 0.516969 & 0.594543 & 0.412089 \\
\hline \hline
\end{tabular}

by performing the computations for all the other values of $w, s$ and $z$. For $A=\{a, b, c, d\}$ and for all pairs $\left(w, w^{B}\right)$ of scoring rules, when $d$ is removed,

$$
\begin{aligned}
& P_{\infty}^{I C}\left(\left(w, w^{B}\right), a b c / a b c d\right)=P_{\infty}^{I C}\left(\left(w, w^{B}\right), a b c / d a b c\right) \\
& P_{\infty}^{I C}\left(\left(w, w^{B}\right), a b c / a b d c\right)=P_{\infty}^{I C}\left(\left(w, w^{B}\right), a b c / a d b c\right)
\end{aligned}
$$

We push the analysis further by addressing the following question: what is the probability for each of the collective rankings on $B=\{a, b, c\}$ to appear given that on $A=\{a, b, c, d\}$. We find the following relations for all pairs $\left(w, w^{B}\right)$ of scoring rules when $d$ is removed.

$$
\begin{aligned}
& P_{\infty}^{I C}\left(\left(w, w^{B}\right), c b a / a b c d\right)=P_{\infty}^{I C}\left(\left(w, w^{B}\right), c b a / d a b c\right) \\
& P_{\infty}^{I C}\left(\left(w, w^{B}\right), c b a / a b d c\right)=P_{\infty}^{I C}\left(\left(w, w^{B}\right), c b a / a d b c\right)
\end{aligned}
$$

\footnotetext{
8 We were not able to find a reduced form of this formula. This was also the case for all the other general formulas.
} 
and for $(\phi, \tau)=(a c b, b a c)$ or $(c a b, b c a)$,

$$
\begin{aligned}
& P_{\infty}^{I C}\left(\left(w, w^{B}\right), \phi / a b c d\right)=P_{\infty}^{I C}\left(\left(w, w^{B}\right), \tau / d a b c\right) \\
& P_{\infty}^{I C}\left(\left(w, w^{B}\right), \phi / a b d c\right)=P_{\infty}^{I C}\left(\left(w, w^{B}\right), \tau / a d b c\right) \\
& P_{\infty}^{I C}\left(\left(w, w^{B}\right), \phi / a d b c\right)=P_{\infty}^{I C}\left(\left(w, w^{B}\right), \tau / a b d c\right) \\
& P_{\infty}^{I C}\left(\left(w, w^{B}\right), \phi / d a b c\right)=P_{\infty}^{I C}\left(\left(w, w^{B}\right), \tau / a b c d\right)
\end{aligned}
$$

In Table 4 we report the limiting probabilities for one of the possible collective rankings on $B=\{a, b, c\}$ to appear given that on $A=\{a, b, c, d\}$ for $w=\frac{2}{3}, s=\frac{1}{3}$ and $z=\frac{1}{2}$. More results are provided in Kamwa and Merlin (2015) who used our Maple-Sheet and the above relationships

\begin{tabular}{|c|c|c|c|c|c|c|}
\hline collective rankings & $a b c$ & $a c b$ & $b a c$ & $b c a$ & $c a b$ & $c b a$ \\
\hline$a b c d$ & 0.719518 & 0.138512 & 0.111617 & 0.011626 & 0.011888 & 0.006836 \\
\hline$a b d c$ & 0.840425 & 0.028084 & 0.127008 & 0.002214 & 0.001408 & 0.000858 \\
\hline$a d b c$ & 0.840425 & 0.127008 & 0.028084 & 0.001408 & 0.002214 & 0.000858 \\
\hline$d a b c$ & 0.719518 & 0.111617 & 0.138512 & 0.011888 & 0.011626 & 0.006836 \\
\hline
\end{tabular}
between probabilities in their framework to compute the likelihood of certain voting paradoxes.

Table 4 Probabilities of one of the possible collective rankings on $B=\{a, b, c\}$ appearing given the collective ranking on $A=\{a, b, c, d\}$ for $w=\frac{2}{3}, s=\frac{1}{3}$ and $z=\frac{1}{2}$

\subsection{Optimality: the optimal decision rules}

We have already noted that Gehrlein and Fishburn (1980) were not able to say for which scoring rule the formula they obtained is minimized/maximized; they end with conjectures. Given our approach and general formulas, we can now determine the pair of scoring vectors for which our general formula of consistency is maximized or minimized.

Notice that our general formula is a 3 -variable function; so, the optimization package of MAPLE can be used for this task. We use Algorithm 1 for the maximization and Algorithm 2 for the minimization.

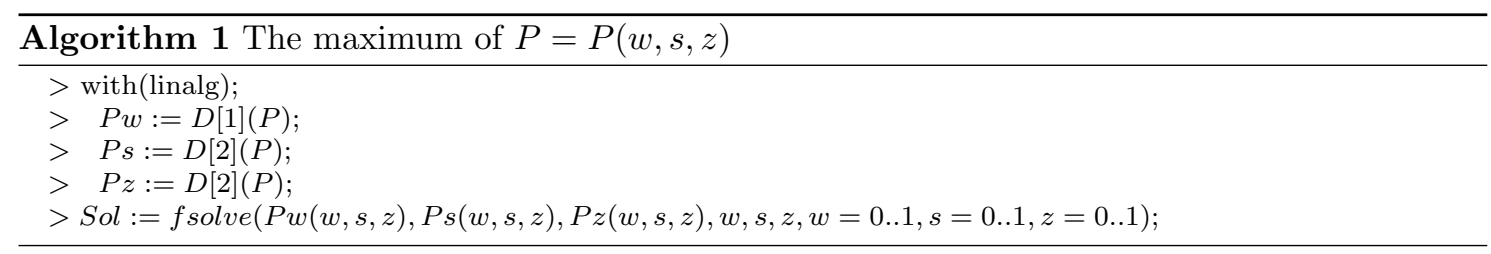

Running Algorithm 1 can be time consuming for some computers. In the end, we get $w=$ $0.6666666, s=0.3333333$ and $z=0.5$ as the solutions of our maximization. These solutions characterize the pair of vectors $w=\left(1, \frac{2}{3}, \frac{1}{3}, 0\right)$ and $w^{B}=\left(1, \frac{1}{2}, 0\right)$. So, we conclude that the consistency probability is maximized by the Borda rule.

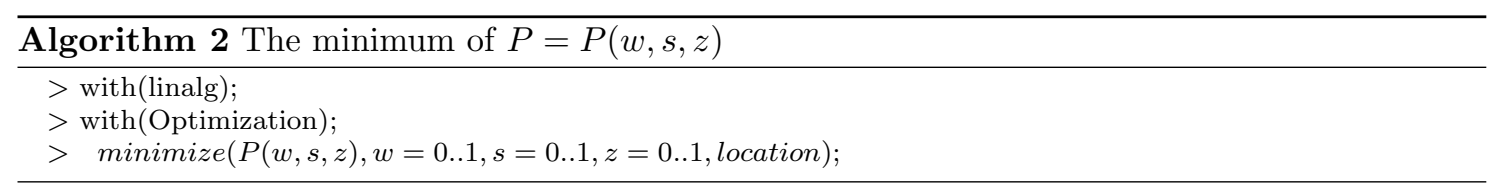


Running Algorithm 2 is time consuming and needs a lot of memory; So the reader will need a robust computer for this. With the help of two members of the MAPLE-group, we found the following solutions: $(w=s=0$ and $z=1)$ and $(w=s=1$ and $z=0)$. So, consistency is minimized with the following pairs of scoring rules :

$$
\begin{aligned}
& \text { (Plurality,Antiplurality })=\left(w=(1,0,0,0), w^{B}=(1,1,0)\right) \\
& \text { (Antiplurality,Plurality) }=\left(w=(1,1,1,0), w^{B}=(1,0,0)\right)
\end{aligned}
$$

Based on all these results, Kamwa and Merlin (2015) were able to derive all their results. They run algorithms similar to Algorithms 1 and 2 and they find that the probabilities of each of the events are minimized by the Borda rule and maximized by the pairs of scoring vectors $\left(w=(1,0,0,0), w^{B}=(1,1,0)\right)$ and $\left(w=(1,1,1,0), w^{B}=(1,0,0)\right)$.

\section{Conclusion}

In this paper, we have applied the Saari-Tataru's technique to a problem already analyzed by Gehrlein and Fishburn (1980): Given a set of four alternatives, how often is a collective ranking on this set consistent with a collective ranking on one of its three-alternative proper subsets. They based their approach on the representation of quadrivariate positive orthants but they were not able to say with certitude what is the optimality of their search. In this paper, we revisit their problem and provide a way to find the optimal decision rule. Further, we provided a Maple-Sheet for the computation of any four-alternative event described by five constraints when scoring rules are used. It could be interesting to see how our proposal can be applied to real-world data as in the papers of Gormley and Murphy (2008) or D'Ambrosio and Heiser (2016) who applied their proposal on a real data set coming from an opinion poll (conducted by the Irish Marketing Surveys in 1997) in which each respondent had to place in order of preference five candidates to be elected President of the Irish Republic. ${ }^{9}$

Acknowledgements The authors are grateful to an anonymous reviewer for his helpful comments. Thanks to two anonymous members of the Maple-group for their assistance. Eric Kamwa acknowledges the support of the Hitotsubashi Institute of Economic Research during his stay under the "JSPS Program for Advancing Strategic International Networks to Accelerate the Circulation of Talented Researchers". Vincent Merlin acknowledges support from the project ANR-14-CE24-0007-01 CoCoRICo-CoDec.

\section{Appendix}

For space constraints, here are the links to:

- An external sheet where the reader can see how our formulas look.

https://www.dropbox.com/s/mkdbxuwfrsevmk3/general\%20formula.pdf?dl=0

- The MAPLE-sheet we used for all our computations.

https://www.dropbox.com/s/byyvtoex90h4je5/MapleSheet_generic.pdf?dl=0

\footnotetext{
9 Thanks to the referee for bringing to our attention the works of D'Ambrosio and Heiser (2016), Gormley and Murphy (2008).
} 


\section{References}

Abrahamson I.G. (1964). Orthant Probabilities for the Quadrivariate Normal Distribution. The Annals of Mathematical Statistics 35(4): 1685-1703.

Berg S, Lepelley D. (1994). On probability models in voting theory. Statistica Neerlandica 48: 133-146.

Coxeter H.S.M. (1935). The functions of Schläfli and Lobatschefsky. Quarterly Journal of Mathematics 6: 13-29.

D'Ambrosio A., Heiser W.J. (2016). A recursive partitioning method for the prediction of preference rankings based upon kemeny distances. Psychometrika 81(3): 774-794.

Felsenthal D.S. (2012). Review of Paradoxes Afflicting Procedures for Electing a Single Candidate in Electoral Systems : Paradoxes, Assumptions, and Procedures, Studies in Choice and Welfare, Felsenthal, DS and Machover, M. (Eds.), Springer Berlin Heidelberg.

Gehrlein W.V (1979). A representation for quadrivariate normal positive orthant probabilities. Journal: Communications in Statistics-simulation and Computation 8(4): 349-358.

Gehrlein W.V. (2006). Condorcet's Paradoxes. Springer Publishing, Berlin.

Gehrlein W.V, Fishburn P.C (1976). The probability of the paradox of voting: A computable solution, Journal of Economic Theory 13: 14-25.

Gehrlein W.V., Fishburn P.C. (1980). Robustness of positional scoring over subsets of alternatives, Appl. Math. Optim. 6: 241-255.

Gehrlein W.V., Fishburn, P.C. (1981). Scoring rules and majority agreements for large electorates with arbitrary preferences, Mathematical Social Sciences 2: 23-33.

Gehrlein, W.V., Lepelley D. (2010). Voting paradoxes and group coherence. Springer.

Gehrlein, W.V., Lepelley, D. (2017) Elections, Voting Rules and Paradoxical Outcomes. Publisher: Springer-Verlag.

Gormley I. C., Murphy T. B. (2008). A mixture of experts model for rank data with applications in election studies. The Annals of Applied Statistics 2(4): 1452-1477.

Kamwa E, Merlin V. (2015). Scoring Rules over Subset of Alternatives : Consistency and Paradoxes, Journal of Mathematical Economics 61: 130-138.

Kellerhals R. (1989). On the volume of hyperbolic polyedra, Mathematische Annalen 285: 541-569.

Kendall, M. G. (1938). A new measure of rank correlation. Biometrika, 30(1/2), 81-93.

Mallows, C. L. (1957). Non-null ranking models. I. Biometrika, 44(1/2), 114-130.

Merlin V, Tataru M, Valognes F. (2002). On the Likelihood of Condorcet's Profiles, Social Choice and Welfare 19(1): 193-206.

Merlin V, Tataru M, Valognes F. (2000). On the probability that all decision rules select the same winner, Journal of Mathematical Economics 33: 183-207.

Merlin V, Valognes F. (2004). On the impact of indifferent voters on the likelihood of some voting paradoxes, Mathematical Social Sciences 48: 343-361.

Milnor J. (1982). Hyperbolic Geometry: the first 150 years, Bull AMS 6: 9-24.

Nurmi H. (1999). Voting Paradoxes and How to Deal with Them. Springer-Verlag.

Plackett R.L. (1954). A Reduction Formula for Normal Multivariate Integrals. Biometrika, 41 $(3 / 4), 351-360$.

Saari D.G, Tataru M. (1999). The likelihood of dubious election outcomes, Economic Theory 13: 345-363.

Schläfli L. (1950). Theorie der Vielfachen Kontinuität. Gesammelte Mathematische Abhandlungen 1. Birkhäuser, Basel.

Tataru M, Merlin V. (1997). On the relationship of the Condorcet winner and positional voting rules, Mathematical Social Sciences 34: 81-90. 